\title{
TECNOLOGÍAS DE LA INFORMACIÓN Y GESTIÓN DE CALIDAD PARA FORTALECER LA EDUCACIÓN CÍVICA CIUDADANA
}

\author{
INFORMATION TECHNOLOGIES AND QUALITY MANAGEMENT TO \\ STRENGTHEN THE CIVIC EDUCATION
}

Jannet Yajaira Velarde Guevara

Bachiller en Ciencias de la Comunicación de la Universidad Nacional Pedro Ruiz Gallo de Lambayeque, Perú

Email: jvelardeg@yahoo.com (Autor Corresponsal)

[Recibido: 21/05/2016 Aceptado: 19/08/2017]

\section{RESUMEN}

El artículo desarrolla un tema de interés para académicos e investigadores como es el de las tecnologías de la información y comunicación así como la gestión de calidad. El tema central es el uso que hacen las instituciones públicas de las tecnologías de la información y comunicación, y cómo estas rigen su desarrollo a través de procesos de calidad. La metodología utilizada comprende el conjunto de aspectos operativos que se tienen en cuenta para realizar el estudio. En esta investigación científica han predominado tres métodos básicos: Inducción, análisis y síntesis. Las principales conclusiones que aquí se presentan, demuestran primero la importancia que tiene hoy en día el uso de las tecnologías de la información y comunicación, y como estos pueden ayudar a procesos y procedimientos establecidos en distintas organizaciones; la gestión de calidad es necesaria porque te permite medir la efectividad e impacto que tienen las tecnologías de la información en pobladores del distrito de Ate en Lima metropolitana, y como se puede replicar en distintos lugares del país. Este artículo es producto del trabajo de maestría del autor, sobre el uso que le dan las instituciones públicas a las tecnologías de la información y comunicación, así como la gestión de calidad que debe estar implícita en sus procesos.

\section{PALABRAS CLAVE}

Tecnologías de la información, gestión de calidad, procesos, efectividad, impacto.

\begin{abstract}
The article develops a topic of interest for academics and researchers such as information and communication technologies, as well as quality management. The central theme is the use that public institutions make of information and communication technologies, and how they govern their development through quality processes. The methodology used includes the set of operational aspects and three basic methods have predominated: Induction, analysis and synthesis. The main conclusions presented here first demonstrate the importance of information and communication technologies use today, and how they can help processes and procedures established in different organizations. Quality management is necessary because it allows the measurement of effectiveness and impact of information technologies on residents of the Ate district in the metropolitan Lima, and how it can be replicated in different parts of the country. This article is the product of the author's work as a teacher, on the use given by public institutions to information and communication technologies, as well as the quality management that should be implicit in their processes.
\end{abstract}

\section{KEYWORDS}

Information technologies, quality management, processes, effectiveness, impact.

Como Citar: Oscanoa, B. (2017). Tecnologías de la información y gestión de calidad para fortalecer la educación cívica ciudadana. Quipukamayoc, 25(49), 91-98 . doi: http://dx.doi.org/10.15381/quipu.v25i49.14284 


\section{INTRODUCCIÓN}

Teniendo en cuenta que hoy en día las tecnologías de la información y comunicación se han convertido en un factor dominante y que se va internalizando en las distintas organizaciones tanto públicas como privadas, convirtiéndose muchas veces en una alternativa de solución, para agilizar y efectivizar procesos dentro de una entidad, cumplir objetivos institucionales, $y$ atender de manera masiva a determinados públicos objetivos o ciudadanos; este artículo presenta el resultado de un trabajo de investigación en el tema de tecnologías de la información, así como gestión de calidad, pues van de la mano en el quehacer institucional.

Cuando se hace referencia al uso de las tecnologías de la información y comunicación en las instituciones públicas, es necesario tener en cuenta para su estudio, la necesidad de medir su efectividad bajo estándares de calidad así como la interrelación de diferentes componentes. Estos son elementos necesarios para aumentar la efectividad e impacto en las personas (Maldonado, 2010).

El objetivo de este trabajo es presentar un análisis acerca de cómo una institución pública hace uso de las tecnologías de la información y comunicación para poder atender las necesidades de sus usuarios, como una alternativa no tradicional; así mismo, podemos mencionar que esto se enmarca en estándares de calidad como el uso del modelo PHVA (planificar, hacer, verificar y actuar), teniendo como unidad de análisis usuarios del subproceso voto informado del Jurado Nacional de Elecciones (Castellanos,2012).

Para conseguir este objetivo, se pre- sentan algunos apartados, así: el primero hace una revisión del concepto de tecnologías de la información y comunicación, como su relación con la gestión de calidad enmarcado en el modelo PHVA. Además se presenta el método utilizado así como las preguntas de investigación y las preposiciones de estudio. También se abordan los resultados de la forma en como las tecnologías de la información y comunicación impactan en determinados usuarios, bajo un modelo de calidad. Por último se plantean las conclusiones así como la discusión del estudio.

Este trabajo pretende aportar en el estudio acerca de las tecnologías de la información y comunicación, y un aporte dirigido al sector público para entender de mejor forma en la práctica ¿cómo bajo estándares de calidad se puede lograr un mejor uso de las tecnologías de la información y comunicación?.

El término tecnologías de información y comunicación (TIC) tiene dos concepciones: por un lado, a menudo se usa "tecnologías de la información" para referirse a cualquier forma de hacer cómputo; por el otro, como nombre de un programa de licenciatura, se refiere a la preparación que tienen estudiantes para satisfacer las necesidades de tecnologías en cómputo y organización.

Planificar y gestionar la infraestructura de TIC de una organización, es un trabajo difícil y complejo que requiere una base muy sólida de la aplicación de los conceptos fundamentales de áreas: las ciencias de la computación y los sistemas de información, así como de gestión y habilidades del personal (Vargas, 2011).

Se requieren habilidades especiales para comprender cómo se componen, estructuran, identificar sus fortalezas, debilidades los sistemas en red.

En sistemas de información se tiene especial cuidado por la fiabilidad, seguridad, facilidad de uso, eficacia y eficiencia del software para los fines previstos, son vitales para cualquier tipo de organización.

Los profesionales en TIC combinan correctamente los conocimientos, prácticas y experiencias para atender tanto la infraestructura de tecnología de información de una organización y las personas que lo utilizan. Asumen la responsabilidad de la selección de productos de hardware y software adecuados para una organización.

Se integran los productos con las necesidades y la infraestructura organizativa, la instalación, la adaptación y el mantenimiento de los sistemas de información, proporcionando así un entorno seguro y eficaz que apoya las actividades de los usuarios del sistema de una organización.

En TIC, la programación a menudo implica escribir pequeños programas que normalmente se conectan a otros programas existentes.

El conjunto de recursos, procedimientos y técnicas usadas en el procesamiento, almacenamiento $\mathrm{y}$ transmisión de información, se ha matizado de la mano de las TIC, pues, en la actualidad, no basta con hablar de una computadora cuando se hace referencia al procesamiento de la información.

Internet forma parte de ese procesamiento que posiblemente se realice de manera distribuida y remota. $\mathrm{Al}$ hablar de procesamiento remoto, además de incorporar el concepto 
de telecomunicación, se puede estar haciendo referencia a un dispositivo muy distinto a lo que tradicionalmente se entiende por computadora pues podría llevarse a cabo, por ejemplo, con un teléfono móvil o una computadora ultra-portátil, con capacidad de operar en red mediante una comunicación inalámbrica y con cada vez más prestaciones, facilidades y rendimiento .

Es indudable el rol que cumple las tecnologías de la información y comunicación (TIC) en nuestra vida diaria, no sólo en el aspecto laboral o profesional. En ese sentido, es necesario identificar su desarrollo en la sociedad bajo estándares, modelo o indicadores de calidad (Miranda, 2012).

El modelo de calidad utilizado en este trabajo es el ya conocido ciclo Deming o ciclo PHVA (planificar, hacer, verificar, actuar), bajo este sistema se optó por utilizar esta metodología (ver Figura 1).

En la actualidad, las instituciones tienen que enfrentarse a un nivel tan alto de competencia que para poder crecer y desarrollarse. A veces incluso para lograr su propia supervivencia, han de mejorar continuamente, evolucionar, renovarse de forma fluida y constante. El ciclo PHVA de mejora continua es una herramienta de gestión presentada en los años 50 por el estadístico estadounidense (Deming, 1989).

Tras varias décadas de uso, este sistema o método de gestión de calidad se encuentra plenamente vigente (ha sido adoptado recientemente por la familia de normas ISO) por su comprobada eficacia para: reducir costos, optimizar la productividad, ganar cuota de mercado e incrementar la rentabilidad de las organizaciones.
Logra, mantenimiento de todos estos beneficios de una manera continua, progresiva y constante (Torres 2011). Planificar: En la etapa de planificación se establecen objetivos y se identifican los procesos necesarios para lograr unos determinados resultados de acuerdo a las políticas de la organización. En esta etapa, se determinan también los parámetros de medición que se van a utilizar para controlar y seguir el proceso.

Hacer: Consiste en la implementación de los cambios o acciones necesarias para lograr las mejoras planteadas. Con el objeto de ganar en eficacia y poder corregir fácilmente posibles errores en la ejecución, normalmente se desarrolla un plan piloto a modo de prueba o testeo.

Verificar: Una vez que se ha puesto en marcha el plan de mejoras, se establece un periodo de prueba para medir y valorar la efectividad de los cambios. Se trata de una fase de regulación y ajuste.

Actuar: Realizadas las mediciones, en el caso de que los resultados no se ajusten a las expectativas y objetivos predefinidos, se realizan las correcciones y modificaciones necesarias. Por otro lado, se toman las decisiones $\mathrm{y}$ acciones pertinentes para mejorar continuamente el desarrollo de los procesos (ver Figura 2).

La principal característica de un ciclo PHVA es que no tiene un punto y final en el momento en que se obtenga un determinado resultado, sino que se crea una rueda continua en la que el ciclo se reinicia constantemente de manera periódica y que genera de esta forma un proceso de mejora continua (Crosby, 1995).

Cada ciclo terminado, además de conseguir mejoras hasta un cierto nivel en una determinada área de la institución, debe servir también como fuente de aprendizaje para mejorar en cada paso y aprender de los errores (Ferney, 2017).

Esto significa que siempre se debe buscar la optimización de las acciones por medio del análisis de indicadores, logros obtenidos y programas de mejora ya implementados.

Tal como se hace mención, la relación existente entre gestión de calidad y Tecnologías de la Información, radica en cómo las instituciones tanto públicas como privadas hacen uso de estas TIC sin dejar de lado el desarrollo de procesos que se rigen bajo estándares o modelos de calidad para conseguir metas y lograr cumplir los objetivos institucionales.

\section{MATERIAL Y MÉTODOS}

El trabajo de investigación realizado para este artículo utilizó el estudio de investigación científica predominando tres métodos básicos: Inducción, análisis y síntesis (Bernal, 2010).

Es una investigación cuantitativa, empírica (porque es un hecho que ocurre en nuestra institución) y aplicada porque se va enfrentar el problema proponiendo alternativas de solución. El tipo de investigación es correlacional, sobre la relación entre gestión de calidad y tecnologías de la información " $\mathrm{X}$ " y satisfacción " $Y$ " en el subproceso voto informado del Jurado Nacional de Elecciones, es una investigación cuantitativa, empírica (porque es un hecho que ocurre en nuestra institución) y aplicada porque se va enfrentar el problema proponiendo alternativas de solución (ver Tabla 1). 
La unidad de análisis es el subproceso Voto Informado del Jurado Nacional de Elecciones, como unidad que brinda un servicio educativo a diferentes usuarios de Lima. La población de estudio estuvo conformada por ciudadanos del distrito de Ate correspondiente a 60085 electores, quiere decir, ciudadanos en edad de votar; de lo cual sólo fueron capacitados el $2 \%$ de la población en el año 2016, esto corresponde a 1201 electores.

La muestra de la investigación está conformada por 100 actores electorales del distrito de Ate Vitarte. La selección de la muestra fue estratificada en forma "no probabilística", es decir de selección intencional, eligiendo aquellos elementos que se consideran más representativos (Bernal, 2010). Se delimitó un número de 100 actores electorales los cuales radican en el distrito de Ate, provincia de Lima.

\section{RESULTADOS}

A continuación, se presentan los prin- cipales hallazgos encontrados en la investigación y que aportan o validan la literatura sobre gestión de calidad y TIC (X) y satisfacción de actores electorales $(\mathrm{Y})$.

A fin de demostrar el grado de asertividad entre la gestión de calidad, las TIC y la satisfacción de actores electorales, se utiliza información procesado de una encuesta aplicada a los usuarios del subproceso voto informado utilizando la escala de Likert.

Tabla 1.

Cuadro resumen que revela el interés de los ciudadanos de Ate, por cada una de las actividades del subproceso Voto Informado para fortalecer la educación cívica ciudadana

Fuente: Elaboración propia.

\begin{tabular}{|c|c|c|c|c|c|c|}
\hline VARIABLES & PREGUNTAS DE ENCUESTA & $\begin{array}{l}\text { Totalmente } \\
\text { de acuerdo }\end{array}$ & De acuerdo & $\begin{array}{l}\text { Ni de acuerdo } \\
\text { ni en } \\
\text { desacuerdo }\end{array}$ & Desacuerdo & $\begin{array}{c}\text { Totalmente en } \\
\text { desacuerdo }\end{array}$ \\
\hline \multirow{7}{*}{$\begin{array}{l}\text { GESTIÓN } \\
\text { DE } \\
\text { CALIDAD Y } \\
\text { TIC }\end{array}$} & $\begin{array}{l}\text { ¿La elaboración de un plan nacional } \\
\text { de educación influye en la calidad } \\
\text { del servicio educativo que reciben } \\
\text { los actores electorales? }\end{array}$ & $68 \%$ & $18 \%$ & $10 \%$ & $2 \%$ & $2 \%$ \\
\hline & $\begin{array}{l}\text { ¿Los talleres de capacitación que } \\
\text { realiza el Subproceso voto informa- } \\
\text { do influyen en el servicio educativo } \\
\text { de calidad que reciben los actores } \\
\text { electorales? }\end{array}$ & $48 \%$ & $10 \%$ & $21 \%$ & $12 \%$ & $9 \%$ \\
\hline & $\begin{array}{l}\text { ¿Las encuestas de satisfacción refle- } \\
\text { jan la calidad otorgada en el servicio } \\
\text { educativo del subproceso voto infor- } \\
\text { mado? }\end{array}$ & $32 \%$ & $43 \%$ & $12 \%$ & $7 \%$ & $6 \%$ \\
\hline & $\begin{array}{l}\text { ¿Las medidas correctivas deben im- } \\
\text { plementarse para mejorar la calidad } \\
\text { del servicio educativo del subproce- } \\
\text { so voto informado? }\end{array}$ & $67 \%$ & $28 \%$ & $5 \%$ & $8 \%$ & $2 \%$ \\
\hline & $\begin{array}{l}\text { ¿El servicio educativo de calidad } \\
\text { ofrecido por profesionales debe im- } \\
\text { plicar las capacitación permanente } \\
\text { de éstos, debido a los temas que in- } \\
\text { volucra a la ciudadanía? }\end{array}$ & $59 \%$ & $31 \%$ & $3 \%$ & $2 \%$ & $5 \%$ \\
\hline & $\begin{array}{l}\text { ¿Considera usted necesario que el } \\
\text { servicio educativo que ofrece voto } \\
\text { informado debería darse utilizando } \\
\text { las TIC? }\end{array}$ & $20 \%$ & $43 \%$ & $18 \%$ & $12 \%$ & $7 \%$ \\
\hline & $\begin{array}{l}\text { ¿Considera que las capacitaciones } \\
\text { virtuales, app voto informado, pági- } \\
\text { na web, redes sociales son necesarios } \\
\text { para el subproceso voto informado? }\end{array}$ & $72 \%$ & $23 \%$ & $2 \%$ & $2 \%$ & $1 \%$ \\
\hline
\end{tabular}




\begin{tabular}{|c|c|c|c|c|c|c|}
\hline & $\begin{array}{l}\text { El uso de las TIC ayudaría al ofre- } \\
\text { cer una mejor calidad en el servicio } \\
\text { educativo del subproceso voto infor- } \\
\text { mado }\end{array}$ & $59 \%$ & $40 \%$ & $3 \%$ & $4 \%$ & $4 \%$ \\
\hline \multirow{8}{*}{$\begin{array}{l}\text { SATISFAC- } \\
\text { CIÓN DE } \\
\text { ACTORES } \\
\text { ELECTORA- } \\
\quad \text { LES }\end{array}$} & $\begin{array}{l}\text { ¿Es importante la prontitud en el } \\
\text { servicio educativo otorgado en el } \\
\text { subproceso voto informado? }\end{array}$ & $45 \%$ & $50 \%$ & $2 \%$ & $1 \%$ & $2 \%$ \\
\hline & $\begin{array}{l}\text { ¿El servicio de calidad, de capacita- } \\
\text { ción y talleres otorgado en el subpro- } \\
\text { ceso voto informado cumple con el } \\
\text { tiempo del ciclo del servicio educati- } \\
\text { vo planificado? }\end{array}$ & $5 \%$ & $66 \%$ & $19 \%$ & $4 \%$ & $6 \%$ \\
\hline & $\begin{array}{l}\text { ¿El servicio educativo de calidad, de } \\
\text { capacitación y talleres otorgado en el } \\
\text { subproceso voto informado es exac- } \\
\text { to en su cumplimiento? }\end{array}$ & $3 \%$ & $88 \%$ & $7 \%$ & $1 \%$ & $1 \%$ \\
\hline & $\begin{array}{l}\text { ¿Es necesaria la retroalimentación o } \\
\text { feedback para que se dé un servicio } \\
\text { educativo de calidad en el subproce- } \\
\text { so voto informado? }\end{array}$ & $79 \%$ & $8 \%$ & $6 \%$ & $2 \%$ & $2 \%$ \\
\hline & $\begin{array}{l}\text { ¿A mayor alcance del servicio educa- } \\
\text { tivo del subproceso voto informado, } \\
\text { mayor calidad? }\end{array}$ & $70 \%$ & $14 \%$ & $7 \%$ & $1 \%$ & $1 \%$ \\
\hline & $\begin{array}{l}\text { ¿El cumplimiento de los procedi- } \\
\text { mientos del Subproceso voto infor- } \\
\text { mado significa mejora de la calidad } \\
\text { en el servicio educativo que se ofrece } \\
\text { en dicho subproceso? }\end{array}$ & $49 \%$ & $16 \%$ & $15 \%$ & $12 \%$ & $8 \%$ \\
\hline & $\begin{array}{l}\text { ¿El cumplimiento de gastos es un in- } \\
\text { dicador de la calidad en el subproce- } \\
\text { so voto informado? }\end{array}$ & $2 \%$ & $25 \%$ & $71 \%$ & $1 \%$ & $1 \%$ \\
\hline & $\begin{array}{l}\text { ¿El cumplimiento de metas en el } \\
\text { subproceso Voto Informado signifi- } \\
\text { ca que se ha logrado trabajar con ca- } \\
\text { lidad el servicio educativo que ofrece } \\
\text { dicho subproceso? }\end{array}$ & $67 \%$ & $15 \%$ & $8 \%$ & $5 \%$ & $5 \%$ \\
\hline
\end{tabular}

En base a la información se realizó el contraste y la prueba de hipótesis para su validación científica.

\section{De la hipótesis}

La gestión de calidad y las TIC influye en la satisfacción de los actores electorales del Jurado Nacional de Elecciones.

Se desarrollan los siguientes pasos:

1. Planteamiento:

Ho: La gestión de calidad y las TIC no influye en la satisfacción de los actores electorales del Jurado Nacional de Elecciones.

Ha: La gestión de calidad y las TIC influye en la satisfacción de los actores electorales del Jurado Nacional de Elecciones.

2. Nivel de significancia: (nivel de confianza de 95\%).

3. Criterios para la contrastación: p-valor $<0.05$, se rechaza la Ho y se acepta Ha.

p-valor $\geq 0.05$, se acepta la Ho.

4. Prueba estadística

Mediante el software SPSS Statistics, se calcula la correlación entre las variables gestión de calidad, tecnologías de la información y satisfacción, y el estadístico de análisis de regresión lineal simple, para evaluar dicha relación y estimar una recta de regresión, que nos permita estimar el efecto de una variable sobre otra (Tabla 1 y 2 ). 
Tabla 2.

Resumen del modelo hipótesis principal Fuente: Elaboración propia.

\begin{tabular}{ccccc} 
Modelo & $\mathbf{R}$ & R cuadrado & R cuadrado corregida & Error tipo de la estimación \\
\hline 1 & 0,460 & 0,212 & 0,204 & 0,12072 \\
\hline \multicolumn{2}{l}{ a. Variables predictoras: (Constante), gestión de calidad, las TIC }
\end{tabular}

Tabla 3.

Coeficientes de regresión lineal hipótesis principal Fuente: Elaboración propia.

\begin{tabular}{|c|c|c|c|c|c|c|c|c|}
\hline & \multirow{2}{*}{ Modelo } & \multicolumn{2}{|c|}{$\begin{array}{c}\text { Coeficientes no estandari- } \\
\text { zados }\end{array}$} & \multirow{2}{*}{$\begin{array}{c}\begin{array}{c}\text { Coeficientes } \\
\text { tipificados }\end{array} \\
\text { Beta }\end{array}$} & \multirow{2}{*}{$\mathrm{T}$} & \multirow{2}{*}{ Sig } & \multicolumn{2}{|c|}{$\begin{array}{c}\text { Intervalo de confianza de } \\
95,0 \% \text { para B }\end{array}$} \\
\hline & & B & Error tip & & & & $\begin{array}{l}\text { Límite } \\
\text { inferior }\end{array}$ & $\begin{array}{l}\text { Límite } \\
\text { superior }\end{array}$ \\
\hline \multirow[b]{2}{*}{1} & (Constante) & 0,901 & 0,133 & & 6,800 & 0,000 & 0,638 & 1,164 \\
\hline & $\begin{array}{l}\text { Gestión de } \\
\text { calidad }\end{array}$ & 0,396 & 0,077 & 0,460 & 5,160 & 0,000 & 0,244 & 0,548 \\
\hline
\end{tabular}

Decisión:

En el modelo se observa que el valor obtenido para el coeficiente de correlación es de $0,460(\mathrm{R})$, con el valor $\mathrm{p}=0,000<0,05$, lo cual indica que existe una correlación positiva media entre ambas variables, y los coeficientes de regresión son 0,396 y 0,901 , con un coeficiente de determinación del $21,2 \%$ (R cuadrado) y p-valor $=0,00$ $<0,05$, lo que implica que hay evolución de la gestión de calidad y las TIC en función de la satisfacción, por lo que se debe rechazar la Ho.

Conclusión: La gestión de calidad junto con las TIC influye en la satisfacción de los actores electorales del Jurado Nacional de Elecciones.

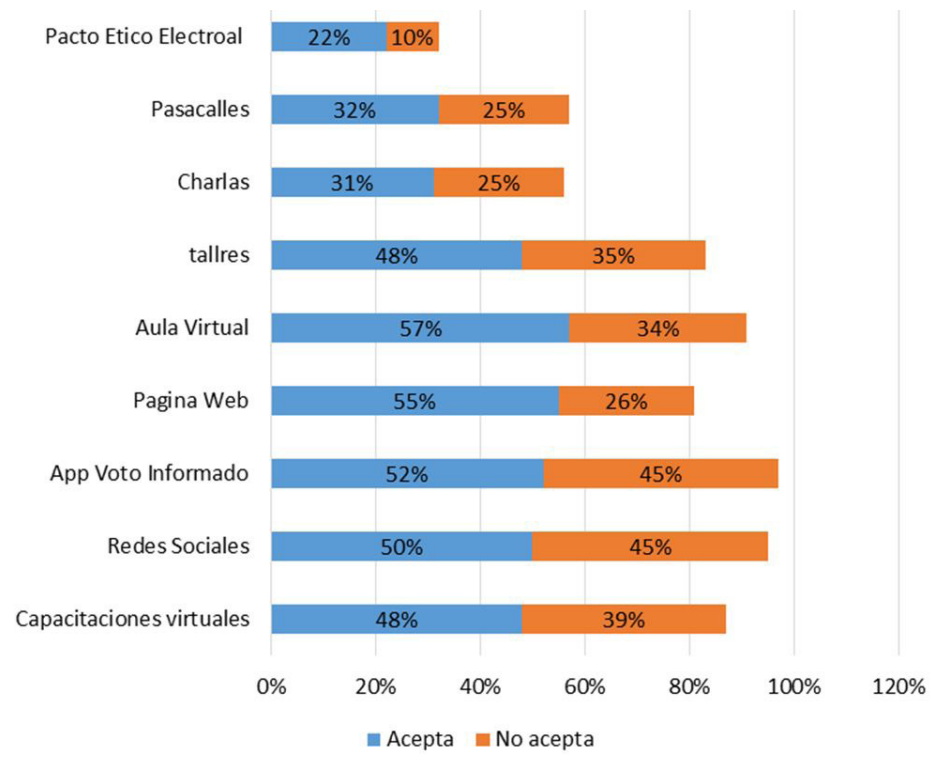

Figura 1. Grado de aceptación y no aceptación que tienen los ciudadanos del distrito de Ate hacia el uso de las tecnologías de la información frente a las actividades tradicionales del subproceso voto informado.

Fuente: Elaboración propia 
Los actores electorales de Ate tienen una mayor aceptación del uso de las tecnologías de la información como por ejemplo capacitaciones virtuales, redes sociales, app Voto Informado, página web, aulas virtuales, sobre una menor aceptación de las actividades tradicionales que son talleres, charlas, pasacalles y el pacto ético electoral desarrollado entre candidatos de un proceso.

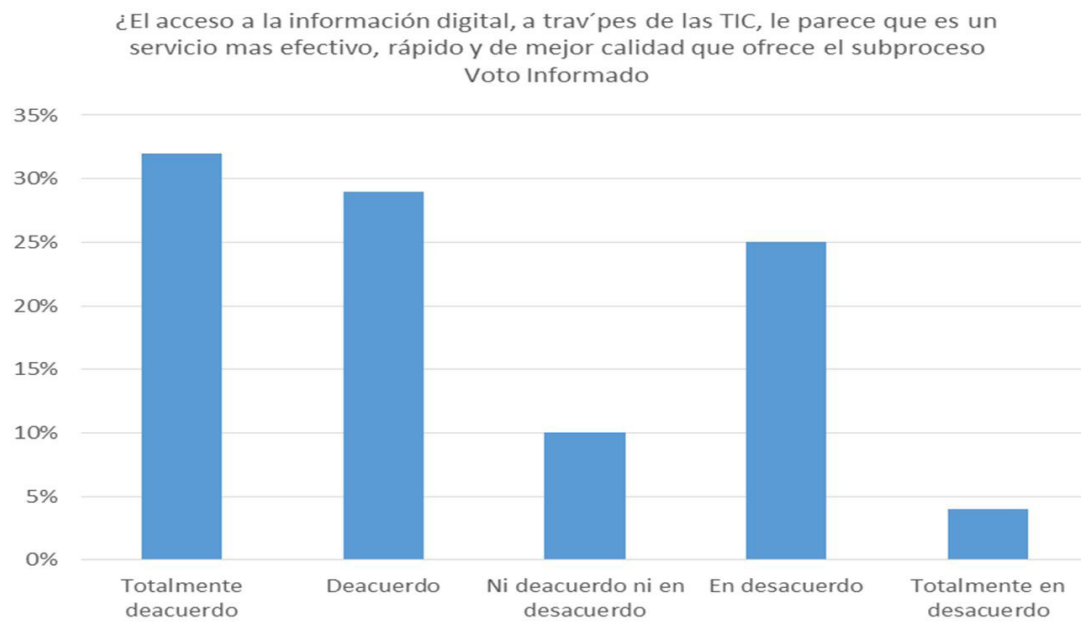

Figura 2. Efectividad, rapidez y calidad en el servicio ofrecido en el subproceso Voto Informado Fuente: Elaboración propia

Al hablar de gestión de calidad, no podemos dejar de mencionar el modelo de Deming o ciclo PHVA: planificar, hacer, verificar y actuar. Este ciclo desarrollado bajo las tecnologías de la Información crean un servicio más efectivo y capaz de llegar a una mayor cantidad de actores electorales. Esta percepción es bien recibida por dichos actores quienes manifiestan estar totalmente de acuerdo en que el servicio educativo ofrecido por el subproceso voto informado es efectivo, rápido y de calidad, haciendo uso de las tecnologías de la información $32 \%$; así mismo, un $29 \%$ señala estar de acuerdo, $10 \%$ ni de acuerdo ni en desacuerdo, 25\% manifiesta estar en desacuerdo y por último un $4 \%$ manifiesta estar totalmente en desacuerdo.

\section{¿Considera Usted necesario que el servicio educativo que ofrece Voto Informado pueda darse utilizando las TIC?}

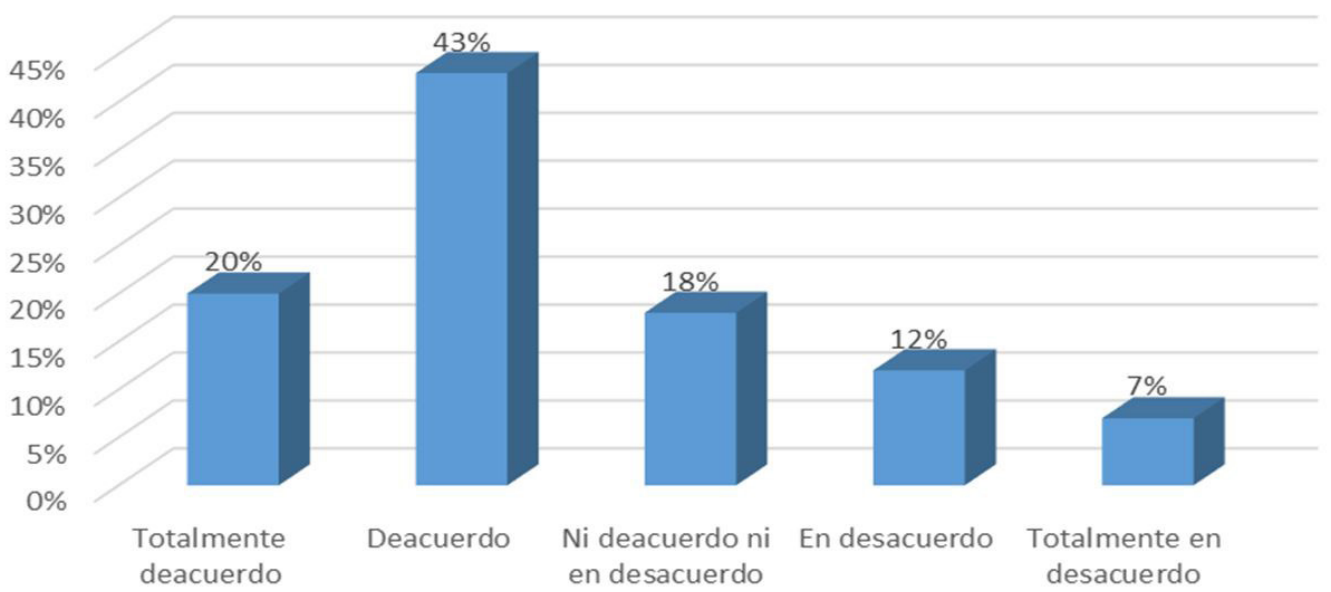

Figura 3. Aprobación de uso de TIC para ofrecer el servicio educativo del sub proceso Voto Informado Fuente: Elaboración propia 
En este último cuadro podemos llegar a la conclusión que existe una aceptación por parte de los actores electorales a que se incluya el uso de las tecnologías de la información de manera permanente, en el servicio que ofrece el voto informado. Un $20 \%$ está totalmente de acuerdo, un $43 \%$ está de acuerdo en la utilización de las TIC en el subproceso Voto Informado, un $18 \%$ señala que no está ni de acuerdo ni en desacuerdo, un $12 \%$ señala que está en desacuerdo y un $7 \%$ señala que está totalmente en desacuerdo.

La importancia que tienen actualmente las tecnologías de la información repercute en las instituciones del estado, y de los ciudadanos para hacer más efectivo el servicio que ofrece, pues haciendo uso de las TIC, nos aseguramos que la información llegue al instante, así como que el mensaje llegue tal cual queremos que llegue, además que permite la interacción entre ciudadanos y estado.

\section{DISCUSIÓN}

El ciclo PHVA, o también conocido como ciclo Deming, es un modelo gerencial que nos permite poder pla- nificar actividades en una empresa o institución pública, además nos permite desarrollar dichas actividades planificadas, también es posible verificar que las actividades que se están ejecutando, se desarrollen conforme a lo planificado y por último también nos permite formular acciones correctivas, para mejorar dicho ciclo (Cuatrecasas, 2010).

El Jurado Nacional de Elecciones a través de su subproceso voto informado cumple con el desarrollo del ciclo PHVA, sin embargo podemos señalar que este subproceso tiene como fin la capacitación electoral, cívico ciudadana, de los ciudadanos, en este sentido se busca fortalecer el "Actuar" del ciclo pues nuestros resultados, existe la necesidad de los ciudadanos que se implementen talleres de capacitación de manera permanente y no solamente en época electoral. Para el Jurado Nacional de Elecciones, como institución pública apunta a través del uso del ciclo Deming, como modelo de Gestión de calidad, a adoptar una visión estratégica que permita dirigir y evaluar el desempeño institucional, especialmente orientado a mejorar la calidad de los productos, consolida estándares que reflejen las necesidades implícitas y obligatorias, proveer información confiable, fortaleciendo de esta manera la educación cívica ciudadana en el Perú (ver Tabla 3).

Ante estas situaciones y realidades que se presentan en diversas entidades públicas es recomendable utilizar las tecnologías de información y comunicación, para estandarizar procesos, reducir costos y agilizar dichos procesos, por ejemplo y teniendo en cuenta el subproceso voto informado, cuyo objetivo es lograr una educación cívica ciudadana de calidad, se hace uso de las capacitaciones virtuales, a través de la página web del Jurado Nacional de Elecciones, y de un aula virtual. Las TIC, se fortalecen, pues desarrollan día a día, y es necesario mirarlos como una opción para mejorar nuestros procesos, agilizarlos, reducir costos, tiempo y mejorar la satisfacción de cualquier tipo de usuario (Ríos, 2011). Es importante mencionar que no existe algún conflicto de interés, por apoyo financiero o material por parte de alguna institución pública o privada.

Según lo señalado se puede concluir lo siguiente: 